Lengua y Sociedad, revista de lingüística teórica y aplicada

Vol. 17, n. ${ }^{\circ}$ 1, Lima, enero-junio de 2018, pp. 9-31

https://doi.org/10.15381/lengsoc.v17i1.22360

\title{
Marcadores conversacionales usados por jóvenes de Managua
}

\section{Conversational Markers Used by Youth in Managua}

\author{
Rosa Amanda Mairena Uriarte \\ Universidad Nacional Autónoma de Nicaragua, Managua \\ rosamairena1@gmail.com
}

\begin{abstract}
Resumen
Para este trabajo investigativo, se creó un corpus con entrevistas semidirigidas a partir del cuestionario propuesto por el Proyecto para el Estudio Sociolingüístico del Español de España y América (PRESEEA). Los informantes son jóvenes de 18 a 34 años que han completado su bachillerato, han crecido en el barrio Monseñor Lezcano, uno de los más antiguos del municipio de Managua, y actualmente residen en la misma localidad. A partir de las transcripciones de las grabaciones, se identificó con ayuda del software Word Smith cuáles eran los marcadores conversacionales del discurso oral de los jóvenes, para clasificarlos de acuerdo con la propuesta de Martín y Portolés (1999) en marcadores de modalidad epistémica, marcadores de modalidad deóntica, enfocadores de la alteridad y metadiscursivos conversacionales. Finalmente, se describió las funciones pragmáticas de estas unidades discursivas.
\end{abstract}

Palabras claves: Marcador discursivo, marcador conversacional, discurso espontáneo juvenil, español de Nicaragua, función pragmática.

\begin{abstract}
For this research work, a corpus of semi-guided interviews was created from the questionnaire proposed by the Project for the Sociolinguistic Study of Spanish in Spain and America (PRESEEA). The informants are young people between the ages of 18 and 34 who have completed high school, have grown up in the Monseñor Lezcano neighbourhood, one of the oldest in the municipality of Managua, and currently live in the same locality. From the transcripts of the recordings, the conversational markers of the oral discourse of the young people were identified with the help of Word Smith software, in order to classify them according to the proposal of Martín and Portolés (1999) in markers of epistemic modality, markers of deontic modality, focusers of otherness and conversational metadiscursives. Finally, the pragmatic functions of these discursive units were described.
\end{abstract}

Keywords: Discursive marker, conversational marker, youthful spontaneous speech, Nicaraguan Spanish, pragmatic function. 


\section{Introducción}

En Nicaragua, los trabajos sobre los marcadores discursivos han seguido dos perspectivas: la didáctica y la lingüística. En el caso de la primera orientación, se han elaborado propuestas didácticas para la enseñanza de los marcadores discursivos a estudiantes de secundaria o del primer año en la educación superior, quienes presentan dificultades en la redacción de textos académicos expositivo-argumentativos ${ }^{1}$. Asimismo, otras propuestas didácticas abordan la enseñanza de los conectores en discursos orales formales, particularmente el debate y la mesa redonda ${ }^{2}$.

Por otra parte, desde la perspectiva lingüística se ha estudiado «El uso de dizque en el español de Nicaragua» (Zamora, 2010). Este artículo presenta los valores de este marcador discursivo en el español peninsular desde principios del siglo XIII hasta inicios del siglo xx. Además, se consultó a 50 informantes de distintas partes de Nicaragua, de entre 17 y 60 años, y con diferente nivel educativo, con el objetivo de corroborar si este marcador era conocido, con qué significado o en qué contexto y si lo utilizaban. De acuerdo con los resultados, se observó que el marcador del discurso «dizque» es empleado mayoritariamente por los jóvenes; en cambio, los adultos, aunque lo conocen, no precisan su significado ni formas de uso.

De igual manera, en una investigación sobre la modalidad y la modalización, se ha identificado 27 marcadores del discurso con distintas funciones pragmáticas, entre ellos bueno y entonces con la función de modalizador enfático como estrategia discursiva en el español hablado por jóvenes de Managua. Sin embargo, estas unidades discursivas son valorados como muletillas (Cuadra, 2012).

De acuerdo con la revisión bibliográfica, se ha constado que los estudios sobre marcadores discursivos en Nicaragua se han centrado principalmente en comprobar si los estudiantes aplican este contenido en su producción discursiva oral o escrita, es decir, si hubo apropiación del tema. Por lo tanto, aún es necesaria la realización de estudios acerca de cómo se utilizan los marcadores discursivos en Nicaragua en discursos orales no académicos, como la conversación y las entrevistas semidirigidas. En este sentido, este trabajo pretende contribuir a la descripción de las funciones pragmáticas de los marcadores conversacionales usados por jóvenes de Managua.

1 Algunos es estos son las tesis de Rodríguez (1999) y Canales y Cárcamo (2010).

2 Entre ellos se encuentran las investigaciones de Escobar (2001, 2008 y 2010) y Leal y Chamorro (2013).

10 Lengua y Sociedad 


\section{Marcadores del discurso}

El discurso es el resultado de utilizar unidades lingüísticas en un acto concreto de comunicación, en el que, así como el componente gramatical, es imprescindible el contexto, esto es, el aspecto pragmático. Dentro del discurso, los enunciados se vinculan mediante unidades lingüísticas denominadas marcadores del discurso, por tal razón, tradicionalmente se han estudiado atendiendo a la sintaxis oracional bajo el nombre de «partículas del discurso». Sin embargo, los marcadores del discurso, además de garantizar la cohesión dando unidad al texto, precisan las inferencias de lo comunicado, por ello se ha analizado su papel externo a la función predicativa, es decir, sus funciones pragmáticas.

Calsamiglia y Tusón (2008) definen los marcadores del discurso como: «Piezas lingüísticas que relacionan de forma explícita segmentos textuales, sean enunciados o secuencias de enunciados, estableciendo entre ellos diversas relaciones semánticas» (p. 235). No obstante, no solo la semántica y la gramática dan cuenta de la función de los marcadores del discurso dentro del sistema lingüístico, más recientemente la pragmática ha ofrecido aportes que permiten una definición mucho más abarcadora y, a la vez, más precisa.

De acuerdo con lo anterior, Martín y Portolés (1999) en su concepto de marcador del discurso asumen un criterio pragmático, porque se define a partir de su función:

Los marcadores del discurso son unidades lingüísticas invariables, no ejercen una función sintáctica en el marco de la predicación oracional —son, pues, elementos marginales- y poseen un cometido coincidente en el discurso: el de guiar, de acuerdo con sus distintas propiedades morfosintácticas, semánticas y pragmáticas las inferencias que se realizan en la comunicación. (p. 4057)

Los marcadores discursivos conforman una categoría pragmática, no deben considerarse una categoría gramatical, puesto que los elementos que integran los marcadores del discurso pertenecen a diferentes clases de palabras: conjunciones, adverbios, interjecciones, sustantivos y formas verbales conjugadas. Sin embargo, a pesar de su heterogeneidad, al conformar una categoría, Martín y Portolés (1999) clasifican los marcadores del discurso según sus funciones en: 1) estructuradores de la información, 2) conectores, 3) reformuladores, 4) operadores argumentativos y 5) marcadores conversacionales. Esta clasificación los autores sintetizan en la tabla siguiente. 
Tabla 1. Clasificación de los marcadores discursivos

\begin{tabular}{|c|c|c|}
\hline \multirow{3}{*}{$\begin{array}{l}\text { Estructuradores } \\
\text { de la información }\end{array}$} & Comentadores & pues, pues bien, así las cosas, etc. \\
\hline & Ordenadores & $\begin{array}{l}\text { en primer lugar/ segundo lugar/; por una } \\
\text { parte, etc. }\end{array}$ \\
\hline & Digresores & por cierto, a todo esto, a propósito, etc. \\
\hline \multirow{3}{*}{ Conectores } & Conectores aditivos & además, encima, aparte, incluso, etc. \\
\hline & $\begin{array}{l}\text { Conectores } \\
\text { consecutivos }\end{array}$ & $\begin{array}{l}\text { por tanto, por consiguiente, por ende, en } \\
\text { consecuencia, de ahí, entonces, pues, así, así } \\
\text { pues, etc. }\end{array}$ \\
\hline & $\begin{array}{l}\text { Conectores } \\
\text { contraargumentativos }\end{array}$ & $\begin{array}{l}\text { en cambio, por el contrario, por el contrario, antes } \\
\text { bien, sin embargo, no obstante, con todo, etc. }\end{array}$ \\
\hline \multirow{4}{*}{ Reformuladores } & $\begin{array}{l}\text { Reformuladores } \\
\text { explicativos }\end{array}$ & o sea, es decir, esto es, a saber, etc. \\
\hline & $\begin{array}{l}\text { Reformuladores } \\
\text { rectificativos }\end{array}$ & mejor dicho, mejor aún, más bien, etc. \\
\hline & $\begin{array}{l}\text { Reformuladores de } \\
\text { distanciamiento }\end{array}$ & $\begin{array}{l}\text { en cualquier caso, en todo caso, de todos } \\
\text { modos, etc. }\end{array}$ \\
\hline & $\begin{array}{l}\text { Reformuladores } \\
\text { recapitulativos }\end{array}$ & $\begin{array}{l}\text { en suma, en conclusión, en definitiva, en fin, al } \\
\text { fin y al cabo, etc. }\end{array}$ \\
\hline \multirow{2}{*}{$\begin{array}{l}\text { Operadores } \\
\text { argumentativos }\end{array}$} & $\begin{array}{l}\text { Operadores de refuerzo } \\
\text { argumentativo }\end{array}$ & en realidad, en el fondo, de hecho, etc. \\
\hline & $\begin{array}{l}\text { Operadores de } \\
\text { concreción }\end{array}$ & por ejemplo, en particular, etc. \\
\hline \multirow{4}{*}{$\begin{array}{l}\text { Marcadores } \\
\text { conversacionales }\end{array}$} & $\begin{array}{l}\text { De modalidad } \\
\text { epistémica }\end{array}$ & claro, desde luego, por lo visto, etc. \\
\hline & De modalidad deóntica & bueno, bien, vale, etc. \\
\hline & $\begin{array}{l}\text { Enfocadores de la } \\
\text { alteridad }\end{array}$ & hombre, mira, oye, etc. \\
\hline & $\begin{array}{l}\text { Metadiscursivos } \\
\text { conversacionales }\end{array}$ & bueno, eh, este, etc. \\
\hline
\end{tabular}

Fuente: Martín y Portolés (1999, p. 4080).

De esta clasificación, en este trabajo se analizan los marcadores conversacionales en el discurso de jóvenes de Managua para estudiar la tendencia a la polifuncionalidad de estas unidades discursivas, pues reflejan las operaciones de estructuración 
de la conversación, las estrategias de cortesía positiva o negativa que señalan el enfoque o posición del hablante respecto al interlocutor, así como las actitudes del hablante en relación con el contenido de los mensajes que se intercambian.

Como se observa en la Tabla 1, los marcadores conversacionales se organizan en cuatro subtipos: 1) marcadores de modalidad epistémica, 2) marcadores de modalidad deóntica, 3) enfocadores de la alteridad y 4) marcadores metadiscursivos. Según Martín y Portolés (1999), los primeros se utilizan en enunciados declarativos y ellos mismos constituyen una aserción que refleja cómo enfoca el hablante el mensaje que el marcador introduce o en el que comparece, según dicho mensaje se considere evidente o conocido a través de otro. A diferencia de estos, los marcadores de modalidad deóntica reflejan actitudes del hablante relacionadas con la expresión de la voluntad o de lo afectivo, es decir, indican si el hablante acepta, admite, consiente en, etc., o no lo que se infiere del fragmento del discurso al que relacionan (pp. 4141-4170).

Por su parte, los enfocadores de la alteridad apuntan, en su origen, fundamentalmente al oyente y, en alguna ocasión, a ambos interlocutores. Sirven para comentar el fragmento del discurso al que remiten, para mostrar la actitud del hablante respecto de este, pero, sobre todo, para señalar el enfoque de las relaciones con el interlocutor, por ello son indicadores de la cortesía verbal. Los marcadores metadiscursivos conversacionales forman parte de los procedimientos que los interlocutores utilizan construir la conversación, para formular e ir organizando su discurso. Se hallan claramente vinculados con los estructuradores de la información y con los reformuladores. Los metadiscursivos conversacionales participan de la función fática del lenguaje, puesto que su objetivo esencial es regular el contacto entre los hablantes (Martín y Portolés, 1999, pp. 4171-4191).

\section{Metodología}

Para la determinación de la muestra de informantes, en este trabajo se retomaron los criterios siguientes: que los hablantes hayan nacido en la ciudad de Managua o, en su defecto, hayan llegado a ella antes de cumplir los diez años o llevaran viviendo más de veinte años específicamente en el barrio Monseñor Lezcano (uno de los más antiguos del municipio de Managua) y que fueran jóvenes de 18 a 34 años, con el bachillerato completo. 
Para la recopilación del corpus se utilizó el cuestionario del Proyecto para el Estudio Sociolingüístico del Español de España y América (PRESEEA), el cual está organizado en nueve módulos temáticos: 1) saludos, 2) el tiempo, 3) lugar donde vive, 4) familia y amistad, 5) costumbres, 6) peligro de muerte, 7) anécdotas importantes en la vida, 8) deseo de mejora económica y 9) final. La selección de este tipo de instrumento obedeció a la naturaleza del objeto de estudio, porque las respuestas son abiertas y se sigue un modelo conversacional. Las entrevistas semidirigidas fueron hechas entre 2015 y 2017. En el presente estudio, se analizan 4 grabaciones, con duración total de 01:56:46.

\section{Marcadores conversacionales en el discurso de jóvenes de Managua}

\subsection{Análisis de los marcadores discursivos de modalidad epistémica}

Entre los marcadores de modalidad epistémica propuestos por Martín y Portolés (1999), se encuentran los marcadores de evidencia: en efecto, efectivamente, desde luego, por supuesto, naturalmente, claro y sin duda; y los marcadores orientativos de la fuente del mensaje: por lo visto, por lo que se ve, al parecer, según parece y a lo que parece. De estos, solamente se registró claro, con una frecuencia total de 2.

(1) E: [Carraspeo] Bueno $\rightarrow$ y en cuanto a los deseos de mejora económica $\rightarrow$ / ¿vos jugás la lotería o algún otro juego de azar? $\uparrow$

I: No pues la verdad es no $\downarrow /$ no no juego porque como digo yo que no tengo suerte $\rightarrow$ / entonces no la compro $\rightarrow$ / entonces $\rightarrow$ / (Risas) claro que me la quisiera sacar pero no la compro $\downarrow$ (Risas) [Inf. M1_02 (170)]

(2) E: Y en general $/$ ¿cómo te gusta que te traten $\uparrow$ de vos o de usted? $\uparrow$

I: De vos $\downarrow / /$ claro que si un chavalito chiquito me dice vos no me gusta $\downarrow$ porque es mala educación $\downarrow$ [Inf. M1_o1 (14)]

El marcador discursivo claro únicamente fue empleado en posición interior de enunciado. Además, aparece acompañado de la conjunción que. En (1) claro 
se emplea como reforzador de una aserción tras una pausa luego de una risa que podría considerarse como estrategia de cooperación e incluso camaradería con la entrevistadora. Además, tematiza con que la aserción de la informante: claro que me la quisiera sacar, referida a la lotería.

En el caso de (2), se observa que claro posee un valor concesivo. Este marcador discursivo permite introducir una autocorrección, es decir, claro anticipa y tematiza con que una objeción ante lo expresado previamente por la misma informante. Por lo tanto, se trata de una estrategia de cortesía que atenúa su rechazo a la forma de tratamiento vos si proviene de alguien menor que ella.

Por otra parte, aunque originalmente no está incluido en la clasificación de Martín y Portolés (1999), se considera la verdad un marcador discursivo de modalidad epistémica. De acuerdo con Serrano (1997) «(...) la verdad pone de manifiesto la realización del contenido asertivo del segmento. (...) exhibe la intención informativa del hablante y su deseo de introducir, bien sea en las respuestas o a lo largo de una secuencia, su implicación afirmativa» (pp. 269-270). Este valor proviene de dos funciones: introductor de respuesta y apoyo a la información.

En el corpus, el marcador discursivo la verdad se registró con una frecuencia de 22 apariciones y fue utilizado en posición inicial, interior y final para indicar el grado de certeza del hablante sobre lo dicho.

(3) E: ¿Y te gusta vivir aquí? $\uparrow$

I: $\quad$ La verdad este $\rightarrow$ en lo general a mí no me gusta Managua $\downarrow$ / es decir $\rightarrow /$ no $\downarrow$ no me gusta $\downarrow$ [Inf. H1_03 (63)]

(4) E: ¿Y has $\rightarrow$ ? ¿te has dado cuenta de que hay un cambio en el clima en estos últimos años? $\uparrow /$ ¿qué opinás al respecto? $\uparrow$

I: $\quad \mathrm{Mmmm} \rightarrow / /$ pues como $\rightarrow /$ bueno $\downarrow$ la verdad es que $\rightarrow$ no le tomo importancia porque como $\rightarrow$ / aquí en la casa con abanico y en el en el trabajo con aire $\rightarrow$ / entonces casi $\rightarrow$ [Inf. M1_O2 (32)]

(5) E: Y del robo $\rightarrow$ ese que sufrió tu hermano $\rightarrow$ ¿qué recordás vos? $\uparrow /$ ¿cómo lo recordás? $\uparrow$

I: $\quad ¡ A h ! \downarrow /$ fue hace poco este $\rightarrow / /$ la verdad es que solo vino alterado $\downarrow /$ le pasóó $\rightarrow$ a unas cuantas cuadras de acá $\downarrow / /$ yyy $\rightarrow /$ yo $\rightarrow$ / y cuando nos comenzó a dar la descripción yo sabía quiénes eran $\downarrow$ (...) [Inf. H1_O3 (121)] 
(6) E: ¿Y cómo te gustaría que fueran tus vecinos? $\uparrow$

I: $\quad \mathrm{Mmm} \rightarrow$ // pues / no sé // no les cambiaría nada / o sea / porque no los conozco $\downarrow /$ la verdad $\downarrow /$ sinceramente $\downarrow$ [Inf. H1_o4 (78)]

En (3) se observa el uso del marcador discursivo en posición inicial absoluta para contestar la interrogante de la entrevistadora. Al tratarse de una contraexpectativa, la verdad funciona como atenuador para introducir la respuesta del informante: en lo general a mí no me gusta Managua. Asimismo, este marcador discursivo de modalidad epistémica se considera una estrategia de cortesía para conservar la imagen negativa del informante, la cual se refuerza con la expresión: a mí, que introduce su punto de vista.

Por su parte, en (4) también se considera que la verdad aparece en posición inicial. En este caso, antepuesto aparece el marcador discursivo bueno que refuerza el valor atenuante al ofrecer una opinión no esperada acerca de un problema mundial: no le tomo importancia (al cambio climático). Según Briz, Pons y Portolés (2008) una característica sintáctica de la verdad es que, a menudo, en la posición inicial, aparece en combinación con es que: la verdad es que. En el corpus de esta investigación, de las 22 apariciones de la verdad, 11 de estas aparecen combinadas con es que, como se observa en (4) y (5).

En (5) el marcador discursivo de modalidad epistémica la verdad se registró en posición interior, y se utiliza acompañado de es que para focalizar el contenido solo vino alterado. Además de reflejar el compromiso del hablante con la veracidad de lo enunciado, la verdad guía al oyente a una inferencia no esperada: a pesar de que el hermano del informante fue víctima de un robo, no sufrió ningún daño físico.

En (6) se ejemplifica un caso de la verdad en posición final, seguido por el adverbio modal sinceramente, con la finalidad de intensificar ese compromiso del hablante con la franqueza de lo dicho, lo cual constituye una justificación a su opinión de que no cambiaría nada a sus vecinos.

Otro marcador discursivo no incluido en la clasificación de Martín y Portolés (1999), pero que marca evidencia es obviamente. De acuerdo con Fuentes (2009), «es un evaluativo de evidencia en el más alto grado. Implica certeza y reafirmación de algo sabido por todos» (p. 242). En el corpus en estudio, este marcador discursivo se registró en el corpus únicamente en 1 aparición:

(7) E: Pero sí me imagino que te gustaría sacarte algún premio I: $\quad=$ No $/$ pues sí $\downarrow=$ 


\section{E: =en la lotería $\downarrow=$ \\ I: Obviamente que sí $\downarrow[$ Inf. H1_O4 (344)]}

En (7) obviamente aparece como réplica y se utiliza como marcador de evidencia para ratificar el miembro del discurso expresado por el interlocutor acerca de que si le gustaría sacarse algún premio en la lotería. Con este marcador discursivo, el hablante manifiesta el acuerdo con la entrevistadora, por lo cual se considera una estrategia de cooperación. Además, en este caso, se observa que obviamente tematiza con que al adverbio sí, por lo cual es utilizado para enfatizar su respuesta afirmativa.

Asimismo, otro de los marcadores discursivos de modalidad epistémica es ideay, el cual proviene de la gramaticalización de la estructura sintáctica y de ahí, que originalmente es una frase adverbial de sentido espacial (Quesada, 1996). Este fue utilizado con una frecuencia total de 5 y únicamente se registró en posición media.

De acuerdo con Hernández (2012), quien estudió la variante diay en Costa Rica, se usa como marcador enfático-evidencia. «Con esta función estaría agregando al enunciado al que acompaña información pragmática, también con una fuerte carfa de pespectiva, del tipo: evidentemente, obviamente, naturalmente, lógicamente, etc.» (p. 105).

En (8), se observa que ideay refuerza la opinión de la hablante sobre el esfuerzo propio y la suerte, punto de vista que había sido introducido con el verbo epistémico creer, conjugado en la primera persona del singular en presente de indicativo. En este caso, la informante considera evidente, la relación entre el esfuerzo y los resultados del mismo.

(8) E: Por ejemplo ¿si tenés alguna bendición a tu vida creés que eso es producto de tu esfuerzo o porque es suerte? $\uparrow$

I: Nooo $\rightarrow$ / sí creo que que es producto de $\rightarrow$ / de lo ideay $\downarrow /$ lo que uno cosecha $\rightarrow$ / va a recibir $\rightarrow$ / y este $\rightarrow$ / entonces $\rightarrow$ / sí creo que sí $\downarrow /$ el que el que $\rightarrow$ / del esfuerzo de uno $\downarrow$ [Inf. M1_02 (180)]

De modo similar, en (9) el informante masculino emplea el marcador discursivo ideay para ratificar su conocimiento sobre el uso de las formas de tratamiento de acuerdo con el nivel de confianza entre los hablantes, lo cual constituye una compentecia comunicativa, dominada y asumida de manera convencional por los habalantes del español de Nicaragua, a través de la inferencia del funcionamiento 
de las relaciones interpersonales, pues para referirse a los médicos suele usarse el pronombre usted. Sin embargo, en el caso del informante, por ser estos médicos amigos de ély, por lo tanto, mantener una relación afectiva, cercana, el pronombre que utiliza para tratarlos, como resultaría evidente, es vos:

(9) E: Y cuando vas al médicoo $\rightarrow /$ ¿cómo tratás al médico? $\uparrow$

I: Bueno $\downarrow$ no voy mucho al médico $\downarrow /$ porque $\rightarrow$ resuelvo con $\rightarrow$ con llamadas de $\rightarrow$ amigos doctores pero $\rightarrow$ ideay $\downarrow$ hay nivel de $\rightarrow$ como hay un nivel de confianza $\rightarrow$ / el trato es de vos $\downarrow$ [Inf. H1_03 (18)]

\subsection{Análisis de los marcadores discursivos de modalidad deóntica}

Los marcadores de modalidad deóntica propuestos por Martín y Portolés (1999) son: bueno, bien, vale, definitivamente, okey y venga. De estos, únicamente se registró okey, con frecuencia de 3 . Este marcador discursivo se emplea en posición inicial.

(10) (...) la primera vez que fui a un «night club» / Y LA ÚNICA QUE HE IDo $\downarrow /$ porque no no me gustan los «night club» fue en Dominicana / pues / que lo bonito de esa semana fue que tenía cuatro amigos $\rightarrow$ / que que había conocido en Costa Rica el año anterior $\downarrow / /$ en Costa Rica / inclusive / creían que yo era dominicano $\downarrow / /$ porque solo / coincidí en todas las conferencias con esos amigos $\downarrow$ / y que cuando llego a Dominicana $\downarrow$ / el primer día me dicen que $\rightarrow$ / mirá papá / me dicen / tenés que salir $\downarrow$ / Okey vamos $\downarrow$ / nosotros te vamos a llevar (...) [Inf. H1_O3 (191)]

(11) E: Muchas gracias por tu tiempo $\downarrow$

I: Okey $\downarrow$

En el caso de (10), se recrea un discurso y se utiliza para mostrar anuencia o conformidad ante la propuesta que el interlocutor relata que le hicieron. En (11), el marcador discursivo okey constituye el turno de habla y constituye una estrategia conversacional que se emplea para establecer el acuerdo entre los participantes del cierre del evento comunicativo. 
Marcadores conversacionales usados por jóvenes de Managua

\subsection{Análisis de los marcadores discursivos enfocadores de la alteridad}

Martín y Portolés (1999) proponen como enfocadores de la alteridad los marcadores hombre, bueno, vamos, mira, mire, oye, oiga, formas verbales de segunda persona y apéndices comprobativos. De estos, se identificó el uso de bueno con frecuencia total de 5. En relación con su posición sintáctica, se identificó en posición inicial y media.

La función de bueno como enfocador de la alteridad es reforzar la imagen positiva del emisor, quien realiza una intervención reactiva a lo expresado por el interlocutor, como se ilustra en los casos siguientes:

(12) E: ¿Y has $\rightarrow$ ? ¿te has dado cuenta de que hay un cambio en el clima en estos últimos años? $\uparrow /$ ¿qué opinás al respecto? $\uparrow$

I: $\quad \mathrm{Mmmm} \rightarrow / /$ pues como $\rightarrow$ / bueno $\downarrow$ la verdad es que $\rightarrow$ no le tomo importancia porque como $\rightarrow$ / aquí en la casa con abanico y en el en el trabajo con aire $\rightarrow$ / entonces casi $\rightarrow$ [Inf. M1_02 (32)]

(13) E: Pero por ejemplo $\downarrow$ /cuando laa comunidad / en la Iglesia realizan cuestiones así / = ¿apoyás? $\rightarrow=$

I: $=$ Bueno $\downarrow$ yo no yo no soy= yo no participo en la parroquia $\downarrow / /$ no soy católico $\downarrow[$ Inf. H1_O3 (169)]

En (12), se utiliza el bueno como atenuante de la opinión que tiene la hablante ante el cambio climático, pues contrario a lo esperado como respuesta a la pregunta de la entrevistadora, la informante refiere le presta poca importancia al fenómeno, lo cual podría representar un desacuerdo entre ambas interlocutoras. Además, refuerza la sinceridad de su postura mediante el uso del marcador de modalidad epistémico la verdad (es que).

De igual manera, en (13), mediante el empleo del marcador discursivo bueno, se realiza una atenuación de la respuesta de no participación en las actividades que organiza la iglesia del barrio donde habita informante, debido a su postura religiosa, la cual él interpreta como posible incomodidad frente a la expectativa de la entrevistadora.

Por su parte, en el caso del marcador discursivo mira, en el corpus, se registró la variante con voseo mirá, puesto que Nicaragua se caracteriza por el uso del voseo pronominal y verbal. Este marcador se utilizó con una frecuencia total de 10. 
(14) [El niño tose y la informante le dice: ponete chinelas Ricardo $\downarrow$ que te vas a enfermar $\downarrow$ mirá que sos llorón $\downarrow$ ] [El niño vuelve a toser] [Inf. M1_01 (43)]

En (14), una informante utiliza mirá dirigirse a su hijo. Este enfocador de la alteridad va acompañado de que, el cual tematiza la información que aparece enseguida: sos llorón. Además, a pesar de que se dirige a un niño, mirá funciona como una estrategia de cortesía positiva que la hablante emplea como un recurso para que el niño comprenda a través de un proceso inferencial guiado la razón del mandato ponete las chinelas y lo obedezca.

Asimismo, en (15) se utiliza mirá para responder a la entrevistadora. En este caso, mirá se utiliza con un enunciado declarativo. También, al igual que en (14), mirá constituye una estrategia de cortesía positiva, para atraer al interlocutor hacia su perspectiva, es decir, funciona como mecanismo de acercamiento cordial entre la informante y la entrevistadora, que le permite a la hablante presentar la justificación de su punto de vista sobre que su barrio no es sano.

(15) E: ¿Y se puede decir que el parque es sano? $\uparrow$

I: No $\downarrow$

E: ¿Por qué? $\uparrow$

I: Porque hay muchos la $\rightarrow$ mirá / hay ladrones, hay marihuaneros // y si te pueden robar / te roban y ya $\downarrow$ [Inf. M1_01 (108)]

En relación con la variante con ustedeo mire, como enfocador de la alteridad, esta fue registrada únicamente en 1 ocurrencia.

(16) E: ¿Si vas a preguntarle a alguien $\rightarrow$ / por ejemplo $\rightarrow$ por una calle o por un $\rightarrow$ la hora? $\uparrow$

I: Usted $\downarrow$

E: ¿Cómo la abordarías a esa persona? $\uparrow$

I: ¿Que $\rightarrow$ cómo formulo la pregunta? $\uparrow$

E: Mjú $\downarrow$

I: $\quad$ Eh $\rightarrow$ por ejemplo si le quiero pedir la dirección $\rightarrow$ mire $\downarrow$ ¿será que usted me puede orientar? $\rightarrow$ / y hago referencia a la dirección que busco $\downarrow[$ Inf. H1_03 (16)] 
En (16) el marcador discursivo mire funciona para atraer la atención del interlocutor y establecer el intercambio comunicativo. A diferencia del mirá, mire, por ser una variante con ustedeo, se considera una estrategia de cortesía positiva, porque aunque con la pregunta ¿será que usted me puede orientar? se atenta contra la imagen negativa, esta agresión al territorio personal se atenúa con mire.

Asimismo, dentro de los enfocadores de la alteridad, Martín y Portolés (1999) también incluyen las formas verbales de segunda persona, cuya base léxica se inscribe en el campo semántico de la percepción física, como ver, y en de la percepción intelectual, como fijarse y saber. Estas formas marcan las relaciones interpersonales entre los participantes en la comunicación y admiten variaciones de número y de tratamiento. En el corpus, se registró ves y vea con 1 ocurrencia cada uno.

(17) E: Bueno $\downarrow$ ¿y si sigue la sequía qué conse $\rightarrow$ qué creés que va a suceder? $\uparrow$ I: $\quad$ Pues $\rightarrow$ la principal / repercusión podría decir que es $\rightarrow$ ah $\rightarrow$ ya la la última podría decirte que es la guerra $\downarrow$ / porqueeee $\rightarrow$ al haber una sequía el agua que es un principal recurso entonces $\rightarrow$ todo mundo lo va a querer $\downarrow$ ves $\downarrow$ se va a conseguir / se va a considerar ya el principal recurso y como es $\rightarrow$ / o sea / inevitable para el ser humano entonces luego se va a hacer una guerra $\downarrow[$ Inf. H1_O4 (44)]

(18) E: Y si es una persona mayor $\rightarrow$ un hombre o una mujer al que por ejem por ejemplo le preguntás la hora en la calle $\downarrow$

I: $\quad$ De usted $\downarrow$

E: ¿Y cómo la abordarías a esa persona? $\uparrow$

I: $\quad$ Vea $\rightarrow$ eh $\rightarrow$ ¿qué hora es? $\uparrow$ [Inf. M1_o1 (12)]

En (17) ves se utiliza en modalidad asertiva para llamar la atención del interlocutor hacia lo planteado por el hablante. En este caso, se emplea en posición interior de enunciado y, además, introduce información ampliada que permite llegar a la conclusión: luego se va a hacer una guerra. Por su parte, en (18) vea constituye una forma imperativa para captar la atención del oyente y comenzar el intercambio comunicativo, es decir, sirve para establecer contacto.

Otro de los enfocadores de la alteridad es sabés, el cual se registró únicamente con 1 frecuencia. 
(19) I: (...) creían que yo era dominicano $\downarrow / /$ porque solo / coincidí en todas las conferencias con esos amigos $\downarrow /$ y que cuando llego a Dominicana $\downarrow /$ el primer día me dicen que $\rightarrow$ / mirá papá / me dicen / tenés que salir $\downarrow$ / Okey vamos $\downarrow /$ nosotros te vamos a llevar / y llegamos a un local que decía Club 707 / que yo entro y los amigos me tenían preparados una bienvenida $\downarrow / /$ vos sabés te ponen el el reflector y te dicen este $\rightarrow$ Un saludo a nuestro amigo centroamericano que nos visita y ya te comienzan el vulgareo $\rightarrow / /$ vos no te imaginás $\downarrow$ [Inf. H1_03 (191)]

En (19) el marcador discursivo sabés se usa con la forma de tratamiento vos, que se usa para enfatizar la confianza y cercanía del informante con la entrevistadora, pues está narrando una anécdota, lo cual constituye un tema de conversación íntimo. En consecuencia, el marcador discursivo sabés funciona como una estrategia de cortesía positiva, que permite establece una relación de confidencialidad, a pesar de ser desconocidos.

Por su parte, el marcador discursivo fijate se identificó en 7 ocurrencias. Este aparece en posición media y final. A continuación, se ilustra cada uno de los casos:

(20) I2: ¿Y esta calle estaba pavimentada? $\uparrow$

I: Creo que sí $\downarrow$

I2: ¿ ¿Desde cuando eras chiquita? $\uparrow$

I: No sé [Informante saluda a un vecinito: hola mi amor $\downarrow$ ] no me acuerdo fijate $\downarrow$ [Inf. M1_O1 (50)]

(21) E: Y bueno $\downarrow$ ¿Vos jugás la lotería? $\uparrow /$ ¿o te anotás en alguna rifa? $\uparrow$

I: Sí $\downarrow /$ tengo buena suerte fijate $\downarrow / /$ en mi anterior trabajo me gané una una licua una lavadora $\downarrow /$ con secadora $\downarrow$ [Inf. M1_o1 (81)]

En (20), fijate se emplea en posición final y funciona como una estrategia de cortesía que atenúa la falta de cooperación de la informante con la entrevistadora, justificada en la imposibilidad de recordar lo que se le solicita. Por su parte, en (21) fijate aparece en posición interior de enunciado, seguido de una pausa, inmediatamente después de la cual se presenta una justificación a la aserción tengo buena suerte. Además, como estrategia de cortesía positiva, permite establecer mayor confianza entre las interlocutoras, pues la informante acerca a la entrevistadora hacia ella. 
En el caso de por favor, este se presentó en 2 ocurrencias en posición final de enunciado. Este marcador se utiliza en enunciados que reflejan peticiones, como se observa a continuación:

(22) [Informante da órdenes a su hijo: andá jugá allá Ricardo / por favor $\downarrow]$ [Inf. M1_O1 (294)]

(23) E: ¿Cómo te gustaría que te tratara? $\uparrow /$ ¿de vos o de usted? $\uparrow$ I: De usted por favor $\downarrow /$ [Ruido de motocicletas]

En (22), el marcador discursivo por favor aparece luego de dos imperativos: andá y jugá. En cambio, en el caso de (23) afecta a un miembro del discurso que explícitamente no incluye un verbo, pero que se infiere de lo que el hablante está planteando: me gustaría que me trataras de usted. En ambos casos, por favor funciona como estrategia de cortesía negativa, pues lo que se pretende es atenuar la orden o solicitud.

Por otra parte, aunque no está incluido en la clasificación de Martín y Portolés (1999), se identificó el uso de la variante con voseo imaginate, con 1 aparición.

(24) E: ¿Y qué hizo el hombre? $\uparrow /$ ¿se robó alguna cosa? $\uparrow$

I: (...) ideay / cuál es mi susto $\rightarrow$ / que el mero 31 me dice una compañera de trabajo: Tip Top $\downarrow /$ me dice $\downarrow /$ andá $\rightarrow$ / me dice $\downarrow /$ Vos no $\downarrow /$ estás panzona $\downarrow / /$ vos sos loca si medio me bajó la regla tres gotitas $\downarrow /$ pero se me quitó $\downarrow / /$ andá hacete una prueba de la de farmacia $\downarrow$ / y yo $\rightarrow$ / incrédula $\downarrow / /$ la $\rightarrow$ / me la hice $\downarrow /$ me metí al baño y CUANDO miro las dos rayas $\downarrow /$ y para mayor seguridad $\rightarrow$ / me la hice en sangre $\downarrow$ / porque yo quería tener mi niño $\downarrow$ / y yo estaba en tratamiento $\downarrow / /$ imaginate qué horrible hubiera sido si ese hombre me hubiera violado $\downarrow /$ y hubiera quedado panzona $\downarrow / /$ aunque $\rightarrow$ ahora hay tantos métodos porque / si me hubiera violado / me hubiera bebido la PPMs / para no salir $\downarrow$ / pero no es seguro $\downarrow$ [Ruido de vehículo circulando] / no es seguro $\downarrow$ [Inf. M1_O1 (272)]

En (24) el marcador discursivo imaginate permite establecer una conexión entre la informante y la entrevistadora, porque la hablante pretende acercar hacia su ámbito, es decir, intenta aproximar a su interlocutora hacía sí para lograr ser 
comprendida, lo cual constituye una estrategia de cortesía positiva que permite establecer una relación de confianza y solidaridad por ser hablantes del mismo sexo.

Dentro de los enfocadores de la alteridad, Martín y Portolés (1999) incluyen los denominados apéndices comprobativos, entre los cuales se encuentran: ¿no?, ¿verdad? y ¿eh?, cuya función es conseguir del oyente la corroboración respecto del segmento discursivo al que remiten.

De estos solamente se identificó ¿verdad? con 1 ocurrencia en posición interior de enunciado. Este se utiliza por parte del hablante para obtener una corrobación del oyente, aunque no haya una respuesta de este, como en (25), por lo cual constituye una estrategia de cortesía negativa, pues no se brinda el espacio para el cambio del turno de habla.

(25) I: (..) a mí cuando me vienen con una rifa / yo lo que le digo a la persona que me viene con la rifa es lo siguiente $\rightarrow / /$ ¿vos sabés qué es la esperanza valleciana? $\uparrow /$ le digo yo $\downarrow / /$ no $\downarrow / /$ me traés cien números / le digo yo $\downarrow /$ y es decir y mis probabilidades son de uno / en cien en ganar ¿verdad? $\uparrow / /$ estamos hablandoo del 1 por ciento $\downarrow / /$ ¿cuánto toca invertir? $\uparrow$ tanto $\downarrow /$ me cuesta tanto $\downarrow /$ ¿cuánto puedo ganar? $\uparrow$ tanto // y siempre pierdo // es decir es una manera de zafármelo $\downarrow$ por eso no me gustan los juegos de ese tipo $\downarrow /$ de azar ni ni loterías ni nada de ese tipo $\downarrow$ [Inf. H1_03 (168)]

De igual manera, no está incluido dentro de la propuesta de Martín y Portolés (1999) como enfocador de la alteridad el marcador discursivo ideay, el cual se registró con una frecuencia de 2, únicamente en posición media.

(26) I: Sí $\downarrow$ / quería $\rightarrow$ se quería llevar el televisor $\downarrow$ / y como yo en un momento de desesperación me metí al cuarto $\downarrow /$ y llamé a mi prima $\downarrow /$ y mi prima vino con mi primo $\downarrow$ / y entonces $\rightarrow$ / [Ruido de vehículo circulando] él mismo $\rightarrow$ / él mismo con un cuchillo $\downarrow$ / y él mismo me amenazó $\downarrow /$ y me $\rightarrow$ / y me $\rightarrow$ / y me hizo abrirle $\downarrow /$ pero en ese $\rightarrow$ / en ese momento que yo le abrí $\rightarrow$ / como que me dio valor y medio me agarré con él $\rightarrow$ / pero ya tenía abierto $\downarrow /$ él ya iba $\rightarrow$ / para afuera con el televisor $\downarrow / /$ en eso mi prima $\downarrow /$ se quedó en la esquina porque mi prima vino $\downarrow /$ Joaquina $\downarrow$ / Joaquina $\downarrow /$ Andate $\downarrow /$ le digo $\downarrow /$ andate $\downarrow / /$ ella no me $\rightarrow$ / o 
Marcadores conversacionales usados por jóvenes de Managua

sea $\downarrow$ / ella sabía porque yo la había llamado $\downarrow / /$ ideay $\downarrow /$ se me metió un hombre aquí adentro $\downarrow / /$ y fijate que (es)tuve así mirá $\downarrow /$ por agarrar un Bygon $\downarrow /$ y echárselo en la cara $\downarrow /$ pero me dio $\rightarrow$ remordimiento $\downarrow$ todavía $\downarrow /$ fijate $\downarrow / /$ eso fue horrible $\downarrow$ [Inf. M1_01 (272)]

En (26) se observa que ideay funciona para captar la atención del oyente ante el enunciado asertivo se me metió un hombre aquí adentro, el cual corresponde a la justificación del acto directivo andate. Además, en este caso ideay refleja las relaciones interpersonales, puesto que se emplea para ejemplificar la forma con la cual se dirige a su prima, por la cercanía y confianza.

Asimismo, aunque no está incluido dentro de la clasificación de Martín y Portolés (1999), se puede considerar ¿ya? como marcador discursivo conversacional. Este se registró en 2 ocurrencias, en la cual manifiesta un valor de comprobación del segmento al que aparece pospuesto, similar a ¿verdad? Así, en (27) se observa que se utiliza para apelar al oyente, aunque no se le dé el espacio para que tome el turno de habla.

(27) I: (...) había dejado / estábamos en un $\rightarrow$ / en un tren / eh / íbamos para otra ciudad / entonces había dejado un libro en / eh / eh $\rightarrow$ // ya habíamos sacado las maletas / habíamos salido / pero como ese libro era prestado / entonces / eh / quise meterme pero ya estaba escuchando que estaban cerrando las puertas $\downarrow / /$ entonces mejor lo dejé ahí / a irme quién sabe adónde $\downarrow$ / que a qué ciudad $\downarrow / /$ ¿ya? $\uparrow / /$ entonces mejor lo dejé $\downarrow$ [Inf. H1_O4 (312)]

\subsection{Análisis de los marcadores metadiscursivos}

Los marcadores metadiscursivos forman parte de los procedimientos que usan los interlocutores para construir la conversación. Entre ellos, Martín y Portolés (1999) incluyen: ya, sí, bueno, bien, eh y este. De estos, se registró el uso sí, bueno, eh y este. Precisamente, por tratarse de unidades que participan en la función fática del lenguaje, se utilizan para regular el contacto entre los hablantes.

El metadiscursivo conversacional sí se identificó en el corpus con 1 ocurrencia. Como se ilustra en (28), el marcador discursivo es utilizado de manera monodialógica, en posición media, con la función de permitirle a la hablante la progresión 
discursiva hacia un comentario final para que luego se realice el cambio de turno de habla.

(28) E: ¿Y / por ejemplo / cuando ustedes van a salir en familia / a qué lugares llevan a los niños? $\uparrow$

I: Al Luis Alfonso $\downarrow /$ pero con cuidado $\downarrow /$ si no vamos al Salvador Allende $\rightarrow / /$ sí $\downarrow /$ hay veces es mejor ni salir $\downarrow$ [Inf. M1_o1 (131)]

Por su parte, el marcador discursivo bueno con función metadiscursiva conversacional se registró en posición inicial y media, con una frecuencia total de 40, como se refleja en (29) y en (30), respectivamente:

(29) E: Por ejemplo en Navidad $\rightarrow$ ¿qué actividades realiza la comunidad? $\uparrow$ I: Bueno / liiii $\rightarrow$ / hacen las act $\rightarrow$ hacen lo del Divino Niño $\rightarrow$ /que pues va el que quiere $\downarrow$ / y este y que hacen la la novena al Divino Niño $\downarrow$ / de allí las procesiones $\downarrow$ / esas de la Virgen son en $\rightarrow$ / en Navidad $\downarrow$ / yyyy $\rightarrow$ / nada más $\downarrow$ las procesiones de la Virgen $\rightarrow$ / yyy el Divino Niño $\downarrow$ [Inf. M1_02 (142)]

(30) I: Cuando yo tenía catorceee $\rightarrow$ / bueno $\downarrow$ mi edad entre los trece catorce y quince años $\rightarrow$ / yo iba mucho al parque $\downarrow / /$ jugué básquet $\downarrow / /$ jugué futbol $\downarrow / / /$ entonceees $\rightarrow$ generalmente lo que me pasa es que todos esos tipos que ahora son ladrones me conocen desde esa edad $\downarrow$ / entonces me los he topado $\rightarrow$ / digamos $\downarrow$ / en una calle Yo solo $\downarrow /$ y ellos tal vez $\rightarrow$ un grupo de cinco $\rightarrow$ / seis tipos y no me roban $\downarrow /$ porqueee $\rightarrow$ / es decir $\rightarrow$ vulgarmente me conozco a todos esos ladrones $\downarrow / / /$ entonces entonces $\rightarrow$ / en ese aspecto de haber tenido esa libertad de ir al parque a jugar $\rightarrow$ básquet y jugar fútbol es beneficioso pues $\downarrow /$ para mi seguridad $\downarrow$

En (29) bueno marca la recepción del mensaje y es también señal del cambio del turno de habla. Por su parte, en (30) bueno se emplea para reformular la idea de manera más precisa y hacer progresar la conversación.

En relación con el marcador metadiscursivo conversacional $e h$, este se identificó con una ocurrencia de 71, en posición inicial y media. Con este marcador discursivo el hablante pretende hacerle saber a su interlocutor que mantendrá el turno de habla mientras ajusta la expresión que desea formular, como en (31): 
(31) E: ¿Qué le gust / qué te gustaría a vos que hubiera para mejorar pues / la forma de entretenimiento sana de la población? $\uparrow$

I: $\quad \mathrm{Mmm} \rightarrow$ // podría hacerse tal vez como que campañas eh $\rightarrow$ / para // como campañas familiares que se hagan / no sé / y se les llama a las familias cada vez que se va a hacer una reunión en el parque / y podría decirse / para que se conocieran y así interactúen entre / entre ellos $\downarrow$ [Inf. H1_O4 (132)]

El marcador metadiscursivo conversacional utilizado con mayor frecuencia por los hablantes jóvenes es este, con un total de 97 ocurrencias, en posición inicial y media. Al igual que eh, este indica que el hablante desea mantener el turno de habla. Una característica de este es que puede aparecer pronunciado con alargamiento vocálico, pues el hablante intenta ganar tiempo mientras encuentra la expresión que considera más adecuada para manifestar lo que realmente desea enunciar. En (32), se puede observar el uso de este tanto con alargamiento vocálico como sin este:

(32) E: ¿Y qué recordás vos del barrio cuando eras pequeña $\downarrow$ además de los amigos? $\uparrow$

I: $\quad \mathrm{Mmm} \rightarrow / /$ antes había bastante esteee $\rightarrow / /$ pandilleros $\downarrow /$ entonces venían de otros barrios $\rightarrow$ / de un barrio de acá que le dicen este $\rightarrow$ / Las Vegas y Las Malvinas $\downarrow$ entonces a veces habían $\rightarrow$ / enfrentamientos $\downarrow /$ [Inf. M1_02 (74)]

Asimismo, aunque tampoco está incluido dentro de la propuesta de Martín y Portolés (1999), el marcador discursivo okey, se registró como un metadiscursivo conversacional con una frecuencia de 3 apariciones, en posición media. Al igual que bueno, el valor que esta partícula presenta es rectificativo y autocorrectivo, como se evidencia en (33):

(33) I: =A uno de los ladrones $=$ a uno de los ladrones lo retiraron del robo $\downarrow / / /$ es decir $\downarrow /$ yo no participé en lo de en eso de la vigilancia por dos motivos $\downarrow / / /$ unO $\downarrow /$ no iba a llegar desvelado a la universidad $\downarrow /$ y Dos $\downarrow$ si yo me metía eso $\rightarrow$ era como que OKEY $\downarrow$ / yo tomo el transporte cerca $\downarrow$ de donde están esos vagos $\downarrow /$ ¿qué me puede 
pasar a mí a las seis $\rightarrow$ de la mañana que estoy esperando un bus / si yo estoy solo? $\uparrow /$ y ya $\downarrow[$ Inf. H1_O3 (129)]

Otro marcador metadiscursivo no mencionado por Martín y Portolés es $a h$, el cual se identificó en el corpus con una frecuencia de 14. Este marcador discursivo señala que el hablante desea conservar el turno de habla mientras formula la expresión adecuada para transmitir lo que realmente desea.

(34) E: ¿Y a vos te gusta ir a pasar las vacaciones a Chinandega? $\uparrow$

I: Sí $\downarrow$

E: ¿Por qué? $\uparrow$

I: Porqueee no tenés / no tengo un contacto tan / ah $\rightarrow$ / un contacto diario / pues con ellos y son mis familiares // así que de vez en cuando se quiere verlos $\downarrow$ [Inf. H1_O4 (268)]

\section{Conclusiones}

El total de marcadores discursivos conversacionales identificados en el corpus en estudio fue de 291. De estos, el mayor número corresponde a los metadiscursivos (226), seguido por los enfocadores de alterirdad (33), los marcadores de modalidad epistémica (29) y, en menor cantidad, los de modalidad deóntica (3).

De los marcadores discursivos de modalidad epistémica planteados por Martín y Portolés se identificó claro, la verdad, obviamente e ideay, que es característico del español de Nicaragua. Claro se registró con 2 apariciones; en un caso se emplea con función de reforzador de una aserción y en otro con valor concesivo. En el caso de la verdad se identificó con una frecuencia de 22, la función de este marcador es la de introducir una contraexpectativa y, a la vez, aporta un valor atenuador a la aseveración del hablante. En el caso de obviamente se registró en un caso, con la función de marcador de evidencia para ratificar el miembro del discurso precedente. Por su parte, ideay se registró con frecuencia total de 5. Este marcador discursivo, se utiliza como reforzador de las aserciones que se consideran evidentes.

En el caso de los marcadores discursivos de modalidad deóntica, en el corpus únicamente se identificó el anglicismo okey, con frecuencia total de 3, en posición inicial. Su principal función es mostrar el acuerdo con lo expresado previamente. 
Además, suele utilizarse como una estrategia conversacional para establecer el cierre del evento comunicativo.

En relación con los enfocadores de alteridad, se registró el uso de bueno, mirá, mire, ves, vea, sabés, fijate, por favor, imagínate, ideay, ¿verdad? y ¿ya? En el caso de bueno, se registró en 5 ocurrencias, en intervenciones reactivas, por lo cual este marcador funciona como atenuador de posibles desacuerdos ante respuestas no esperadas. Por su parte, mirá se identificó en 10 casos. Este permite tematizar la información que le sigue y, además, contribuye a establecer una relación de cercanía entre los interlocutores. En cambio, mire se registró únicamente en 1 ocurrencia y se emplea para establecer el contacto entre los interlocutores, pero de manera cortés, puesto que atenúa la posible molestia del oyente al sentirse importunado por el hablante. En relación con los marcadores ves y vea, cada uno se identificó con 1 ocurrencia, ambos se utilizan para captar la atención del interlocutor. Particularmente, ves introduce un comentario permite llegar a una conclusión. Por su parte, vea se usa para dar inicio al intercambio comunicativo, esto es, establecer contacto.

El marcador discursivo sabés se registró en 1 ocurrencia, en la cual se usa acompañado del pronombre vos y constituye estrategia de cortesía positiva para acercar a los interlocutores. El enfocador de alteridad fijate se identificó en 7 ocurrencias. En posición final, se observa que fijate atenúa la falta de cooperación de la informante con la entrevistadora. En cambio, en posición media este marcador refuerza la imagen positiva de la hablante al acercar a las interlocutoras. En relación con el marcador discursivo por favor, este se registró en 2 ocurrencias, únicamente en posición final y su función es la de atenuar una orden o solicitud. También, se identificó el uso de imagínate, con 1 ocurrencia, en posición media, para acercar a las interlocutoras.

En lo concerniente a los apéndices comprobativos, se identificó ¿verdad? y ¿ya? cada uno con 1 ocurrencia, en posición media. Estos se utilizan para apelar al oyente y mantener su atención. Adicionalmente, se registró el marcador discursivo ideay, con una frecuencia total de 2, en posición media, su función es establecer un acercamiento entre las interlocutoras.

De los marcadores metadiscursivos conversacionales, se registró sí, bueno, eh, este, okey y ah. El metadiscursivo conversacional sí se identificó en el corpus con 1 ocurrencia, en posición media, para garantizarle al hablante progresión discursiva. Por su parte, el marcador discursivo bueno, se registró en posición inicial y media, con una frecuencia total de 40 . Este metadiscursivo conversacional marca 
la recepción del mensaje y señala el cambio del turno de habla. Además, se emplea para reformular la idea de manera más precisa y hacer progresar la conversación.

El marcador metadiscursivo conversacional $\mathrm{eh}$, se identificó con una ocurrencia de 71, en posición inicial y media. Su función es informar al interlocutor que se mantendrá el turno de habla mientras ajusta la expresión que desea enunciar. El marcador metadiscursivo conversacional este, presenta un total de 97 ocurrencias, en posición inicial y media, y también indica que se desea mantener el turno de habla. Asimismo, se registró okey, como con una frecuencia de 3 apariciones, en posición media, con valor rectificativo y autocorrectivo del discurso previo. Otro marcador metadiscursivo conversacional es $a h$, que aparece con una frecuencia de 14 y se emplea para señalar que el hablante conservará el turno de habla mientras formula el enunciado. 
Marcadores conversacionales usados por jóvenes de Managua

\section{Referencias bibliográficas}

Canales, Y. \& Cárcamo, B. (2010). Enseñanza de los marcadores del discurso, con énfasis en los conectores discursivos en estudiantes del Año de Estudios Generales, UNAN-León (Tesis de maestría). Universidad Nacional Autónoma de Nicaragua, León.

Cuadra, N. (2012). El uso de la modalidad y la modelización como estrategias discursivas del español hablado por los jóvenes de Managua (Tesis de maestría inédita). Universidad Nacional Autónoma de Nicaragua, Managua.

Escobar, A. (2001). Uso de conectores lógicos en el discurso formal de los estudiantes de IV del INAV, durante la realización de la mesa redonda (Monografía). Universidad Nacional Autónoma de Nicaragua, Managua.

Escobar, A. (2008). Los conectores y operadores de refuerzo argumentativo, elementos que dan fuerza al discurso oral formal: el debate. Recuperado de http://www.unan.edu.ni/feduci/i\%2ocongreso/didacticas\%2oespeciales/ los\%20conectores\%20y\%20operadores\%2ode\%2orefuerzo.pdf

Escobar, A. (2010). Uso de conectores lógicos en el discurso formal de los estudiantes del IV año del Instituto Nacional Autónomo de Villanueva-Chinandega, durante la realización de la mesa redonda. En Cátedra. 14, Managua: unANManagua, 43-60.

Fuentes, C. (2009). Diccionario de conectores y operadores del español. Madrid: Arco/Libro.

Hernández, M. (2012). Usos y funciones de (i)diay en Costa Rica. Káñina Xxxvi, pp. 101-110.

Leal, M. \& Chamorro, M. (2013). Producción de argumentos orales en la realización de la mesa redonda. (Trabajo de Seminario de Graduación). Universidad Nacional Autónoma de Nicaragua, Managua.

Quesada, M. (1996). Nuevo diccionario de costarriqueñismos. Cartago: Tecnológica de Costa Rica.

Rodríguez, M. (1999). Énfasis en el mejoramiento de la composición de un ensayo expositivo-argumentativo, mediante el empleo de los marcadores textuales (Tesis de maestría). Universidad Nacional Autónoma de Nicaragua, Managua.

Serrano, M. (1997). El uso de la verdad y pues como marcadores discursivos de respuesta. Boletín de Filología de la Universidad de Chile, xxxvi, pp. 265-286.

Zamora, Z. (2010). El uso de dizque en el español de Nicaragua. El Nuevo Diario. Recuperado de http://www.elnuevodiario.com.ni/suplemento/nuevoamanecer/58 